\title{
Coexpression of ABCB1 and ABCG2 in a Cell Line Model Reveals Both Independent and Additive Transporter Function ${ }^{\mathbb{}}$
}

\author{
Andrea N. Robinson, Bethelihem G. Tebase, Sonia C. Francone, Lyn M. Huff, Hanna Kozlowski, \\ Dominique Cossari, Jung-Min Lee, Dominic Esposito, Robert W. Robey, \\ and Michael M. Gottesman
}

Laboratory of Cell Biology (A.N.R., B.G.T., S.C.F., L.M.H., H.K., D.C., R.W.R., M.M.G.) and Women's Malignancies Branch (J.-M.L.), National Institutes of Health, Center for Cancer Research, National Cancer Institute, Bethesda, Maryland; and Protein Expression Laboratory, Frederick National Laboratory for Cancer Research, Frederick, Maryland (D.E.)

Received December 27, 2018; accepted April 23, 2019

\begin{abstract}
Although overexpression of multiple ATP-binding cassette transporters has been reported in clinical samples, few studies have examined how coexpression of multiple transporters affected resistance to chemotherapeutic drugs. We therefore examined how coexpression of ABCB1 (P-glycoprotein) and ABCG2 contributes to drug resistance in a cell line model. HEK293 cells were transfected with vector-encoding full-length $A B C B 1, A B C G 2$, or a bicistronic vector containing both genes, each under the control of a separate promoter. Cells transfected with both transporters (B1/G2 cells) demonstrated high levels of both transporters, and uptake of both the ABCB1-specific substrate rhodamine 123 and the ABCG2specific substrate pheophorbide a was reduced when examined by flow cytometry. B1/G2 cells were also cross-resistant to the $A B C B 1$ substrate doxorubicin, the $A B C G 2$ substrate topotecan, as
\end{abstract}

well as mitoxantrone and the cell cycle checkpoint kinase 1 inhibitor prexasertib, both of which were found to be substrates of both $A B C B 1$ and $A B C G 2$. When B1/G2 cells were incubated with both rhodamine 123 and pheophorbide $a$, transport of both compounds was observed, suggesting that $A B C B 1$ and $A B C G 2$, when coexpressed, can function independently to transport substrates. ABCB1 and $A B C G 2$ also functioned additively to transport the common fluorescent substrates mitoxantrone and BODIPY-prazosin, as it was necessary to inhibit both transporters to prevent efflux from $B 1 / G 2$ cells. ABCG2 expression was also found to decrease the efficacy of the $A B C B 1$ inhibitor tariquidar in B1/G2 cells. Thus, $A B C B 1$ and $A B C G 2$ can independently and additively confer resistance to substrates, underscoring the need to inhibit multiple transporters when they are coexpressed.

\section{Introduction}

Overexpression of ABCB1 (P-glycoprotein, encoded by the $A B C B 1$ gene) or $\mathrm{ABCG} 2$ is known to confer resistance to multiple chemotherapeutic drugs, including many targeted therapies under clinical development (Robey et al., 2018). Additionally, these transporters are known to limit oral bioavailability, as both are expressed in the gastrointestinal tract (Thiebaut et al., 1987; Maliepaard et al., 2001; Fetsch et al., 2006). In knockout models, mice deficient in both $A b c b 1 a$ and $A b c b 1 b$ (the murine homologs of human $A B C B 1$, previously known as mdrla and $m d r l b$ ) demonstrate increased oral bioavailability of ABCB1 substrates such as taxol compared with wild-type mice (Sparreboom et al., 1997). Similar results have been found with the ABCG2 substrate sulfasalazine, as mice deficient in $A b c g 2$ had higher plasma drug

This work was supported in part by the Intramural Research Program of the National Institutes of Health, the National Cancer Institute.

The content of this publication does not necessarily reflect the views or policies of the Department of Health and Human Services, nor does mention of trade names, commercial products, or organizations imply endorsement by the U.S. Government.

https://doi.org/10.1124/dmd.118.086181.

S This article has supplemental material available at dmd.aspetjournals.org. levels after oral administration of the drug compared with control mice (Zaher et al., 2006).

It has been demonstrated that both $\mathrm{ABCB} 1$ and $\mathrm{ABCG} 2$ can limit the oral bioavailability of common substrates. Upon oral administration of the mutant BRAF inhibitor vemurafenib, plasma area under the curve values were 1.6-fold higher in Abcb1a/1b-deficient mice and 2.3-fold higher in Abcg2-deficient mice, but were 6.6-fold higher in mice deficient in both transporters compared with wild-type controls (Durmus et al., 2012). Similarly, plasma areas under the curve of the epidermal growth factor receptor/human epidermal growth factor receptor 2 inhibitor afatinib were 4.2-fold, 2.4-fold, and 7-fold higher in Abcg2, Abcb1a/1b, and double-knockout mice, respectively, compared with wild-type mice (van Hoppe et al., 2017), thus suggesting that both transporters contribute to decreased oral bioavailability of substrates.

Additionally, ABCB1 and ABCG2 are coexpressed in the brain capillaries that form the blood-brain barrier (BBB) (Cooray et al., 2002; Fetsch et al., 2006) and serve to keep toxins and some chemotherapeutic agents out of the brain (Robey et al., 2010). Mouse knockout models point to a compensatory and cooperative role for $\mathrm{ABCB} 1$ and $\mathrm{ABCG} 2$ at the BBB. Brain concentrations of the PARP inhibitor rucaparib were increased by 2-fold, 5.2-fold, and 32.6-fold in mice deficient in Abcg2, Abcb1a/1b, or both transporters, respectively, compared with wild-type controls, suggesting a cooperative role for the two transporters at the BBB

ABBREVIATIONS: BBB, blood-brain barrier; CMV, cytomegalovirus; FTC, fumitremorgin; MEF, mouse embryonic fibroblast; PhA, pheophorbide a; VAL, valspodar. 
(Durmus et al., 2015b). ABCB1 and ABCG2 were found to cooperatively exclude the Janus kinase $1 / 2$ inhibitor momelotinib from the brain, as 8 hours after oral administration of the drug, mice deficient in Abcg2, Abcb1a/b, or Abcg2;Abcb1a/b were found to have 6.5-fold, 3-fold, or 48-fold higher brain levels compared with controls (Durmus et al., 2013). This apparent synergism from deleting both of the murine homologs for $\mathrm{ABCB} 1$ and $\mathrm{ABCG} 2$ results from the fact that transport due to $\mathrm{ABCB} 1$ and $\mathrm{ABCG} 2$ is much higher than passive diffusion of the drugs across the BBB (Kusuhara and Sugiyama, 2009; Kodaira et al., 2010). Coadministration of the dual ABCB1/ABCG2 inhibitor elacridar resulted in significantly increased brain levels of the kinase inhibitors tandutinib (Yang et al., 2010), pazopanib (Minocha et al., 2012), and sunitinib (Tang et al., 2010). ABCB1 and ABCG2 are thus major obstacles to overcome when treating brain cancers or metastases to the brain.

$\mathrm{ABCB} 1$ and $\mathrm{ABCG} 2$ are found to be coexpressed in some cancers, particularly leukemia. Wilson et al. (2006) obtained gene expression profiles of 170 pretreated samples of acute myelogenous leukemia by microarray analysis. Using unsupervised clustering, the patients clustered into six groups; the cluster characterized by the highest levels of resistant disease showed increased expression of ABCB1 and ABCG2 (Wilson et al., 2006). Profiling 380 drug-resistancerelated genes in a set of 11 paired samples obtained at diagnosis and again at relapse identified two patients with increases in both ABCB1 and ABCG2 at relapse (Patel et al., 2013). Liu et al. (2018) examined expression of $\mathrm{ABCB} 1, \mathrm{ABCB} 4, \mathrm{ABCC} 1, \mathrm{ABCC} 4$, and $\mathrm{ABCG} 2$ in bone marrow mononuclear cells from 96 de novo acute myelogenous leukemia patients and found that coexpression of multiple transporters was associated with worse prognosis. Expression of multiple transporters may therefore confer greater resistance to chemotherapy than expression of a single transporter.

Despite evidence suggesting a cooperative and potentially compensatory role for ABCB1 and ABCG2, few studies have addressed how these transporters might be working together to render chemotherapy less effective. We thus generated HEK293 cell lines that express both transporters and find that the transporters function both independently and additively to transport substrates.

\section{Materials and Methods}

Chemicals. Doxorubicin, mitoxantrone, paclitaxel, etoposide, and rhodamine 123 were purchased from Sigma-Aldrich (St. Louis, MO). SN-38 and topotecan were from LKT Laboratories (St. Paul, MN). Valspodar (VAL) was obtained from Apex Biotechnology (Houston, TX). Pheophorbide a $(\mathrm{PhA})$ was purchased from Frontier Scientific (Logan, UT). Zeocin was from InvivoGen (San Diego, CA), and prexasertib from Selleck Chemicals (Houston, TX). BODIPY-prazosin was obtained from Life Technologies (Eugene, OR). Tariquidar was a gift of Xenova Group (Slough, UK). Fumitremorgin (FTC) was synthesized by the National Institutes of Health Chemical Biology Laboratory (Bethesda, MD).

Cell Lines. Human embryonic kidney cells (HEK293) were obtained from American Type Culture Collection (Manassas, VA) and cultured in Eagle's minimum essential medium (Gibco/Life Technologies, Carlsbad, CA) with $10 \%$ FBS, $1 \%$ glutamine, and $1 \%$ penicillin. Cells were transfected with empty vector with zeocin resistance alone, vectors encoding full-length human $A B C B 1$ or $A B C G 2$, or a bicistronic vector in which each gene is under the control of a separate cytomegalovirus (CMV) promoter. $A B C B 1$ and $A B C G 2$ expression constructs were generated by Gateway cloning using human cDNA templates. Both genes were cloned with Kozak translational initiation sites prior to the initiator methionine and stop codons at the $3^{\prime}$ end. Genes were amplified by low-cycle polymerase chain reaction using primers with Gateway
A
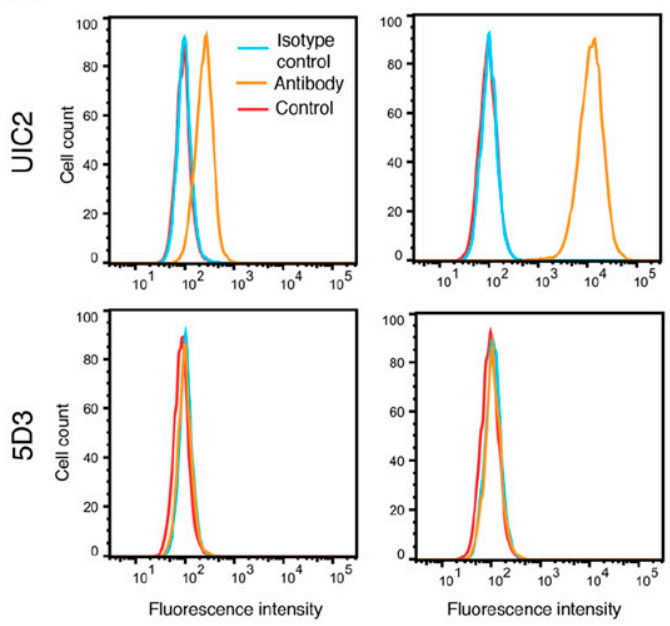

B

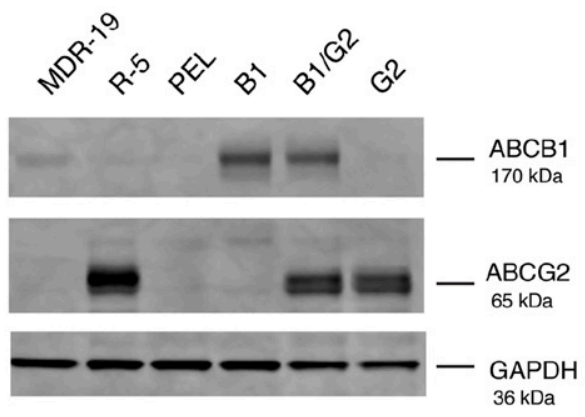

G2
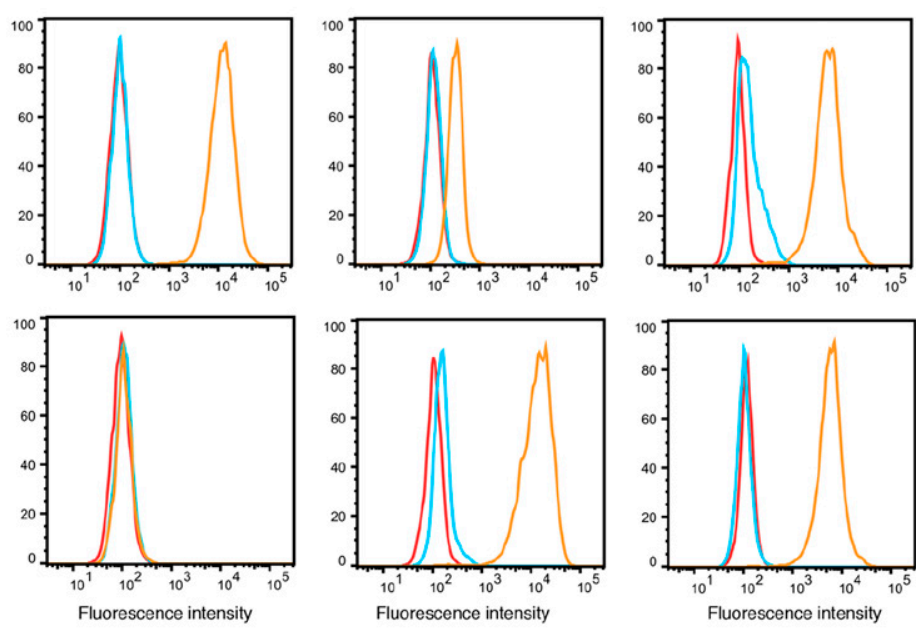

Fig. 1. Characterization of $\mathrm{ABCB} 1$ and ABCG2 expression in transfected HEK293 cells. (A) Trypsinized cells were incubated with phycoerythrin-labeled UIC2 antibody (to detect $\mathrm{ABCB} 1$ ), 5D3 antibody (to detect ABCG2), or the appropriate isotype control for 20 minutes in $2 \%$ bovine serum albumin, after which cells were washed in PBS and read on a flow cytometer. Untreated cells (cell autofluorescence, Control) are denoted by the red curve, cells incubated with the isotype control are denoted by the blue curve, and cells incubated with the specific transporter antibodies are denoted by the orange curve. (B) Wholecell lysates were subjected to PAGE and transferred to a nitrocellulose membrane, which was subsequently probed with antibodies to ABCB1 (UIC2), ABCG2 (BXP-21), and glyceraldehyde-3-phosphate dehydrogenase, which served as a loading control. MDR-19 cells served as a positive control for ABCB1, whereas R-5 cells were a positive control for ABCG2. Near infraredtagged secondary antibodies allowed for protein detection, and band intensity was measured on a LI-COR Odyssey scanner. 


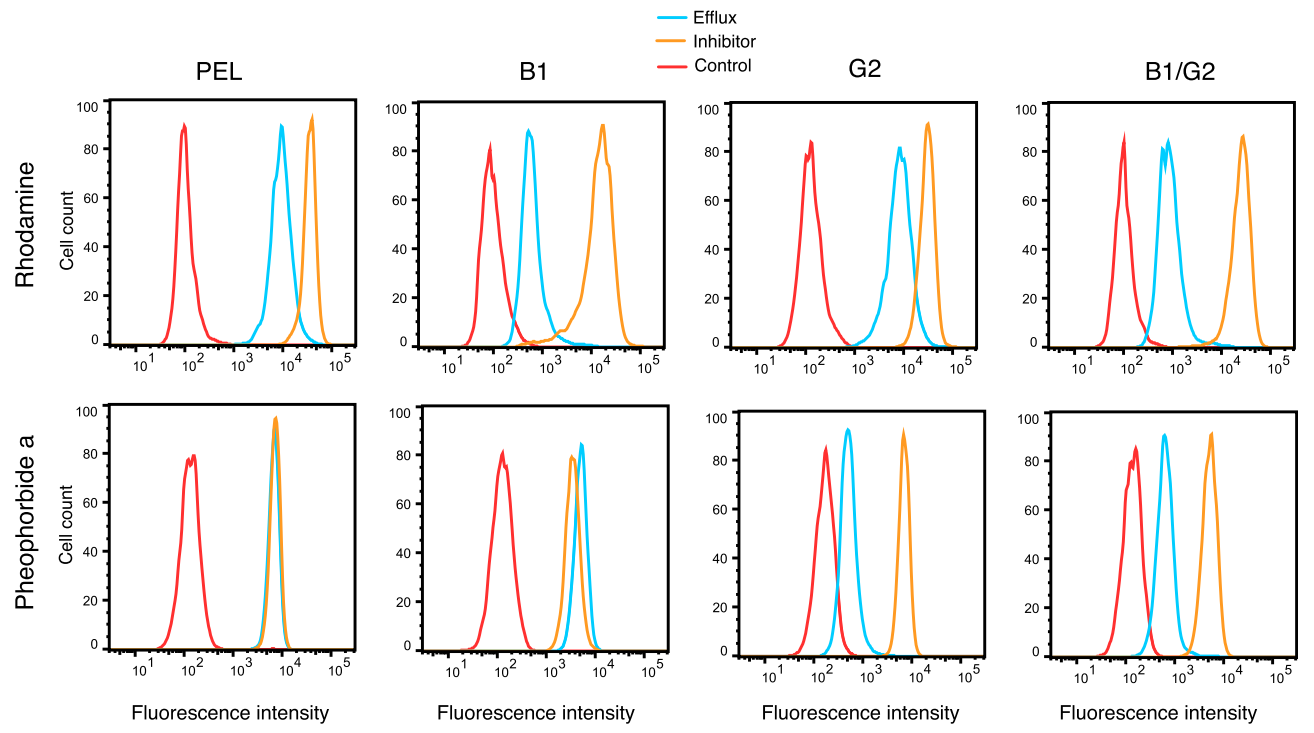

Fig. 2. Characterization of $\mathrm{ABCB} 1$ and ABCG2 function in transfected HEK293 cells. (A) Trypsinized cells were incubated with rhodamine $123(0.5 \mu \mathrm{g} / \mathrm{ml})$ or $\mathrm{PhA}$ $(2 \mu \mathrm{m})$ in the absence or in the presence of a protein-specific inhibitor (3 $\mu \mathrm{M}$ VAL for ABCB1; $10 \mu \mathrm{M}$ FTC for ABCG2) for 30 minutes, after which media was removed and replaced with substrate-free medium continuing with or without the inhibitor for an additional 1 hour. Intracellular substrate fluorescence was measured with a FACSCanto II flow cytometer. Control cells (cell autofluorescence) are denoted by the red curve, cells incubated with the substrate alone are denoted by the blue curve, and cells incubated with the substrate and inhibitor are denoted by the orange curve.

recombination sites, and entry clones were generated using BP recombination under the manufacturer's standard conditions (ThermoFisher, Waltham, MA). Entry clones were fully sequence verified, and the genes were transferred by Gateway LR recombination into pDest-760, a mammalian expression vector based on the pcDNA3.1-zeo vector (ThermoFisher), which contains a moderate-strength CMV promoter and carries a zeocin resistance marker. A control construct was generated in which a stuffer fragment of noncoding DNA was inserted into pDest-760 to serve as a control for protein expression constructs.

A bicistronic $A B C B 1 / A B C G 2$ expression vector was generated using combinatorial Multisite Gateway (Wall et al., 2014) in which $A B C B 1$ was cloned into a Gateway Entry clone flanked by attB4 and attB5 sites. This product was then recombined with a CMV13 promoter fragment flanked with attB5 and attB1 sites and the standard $A B C G 2$ Entry clone previously generated. This recombination was carried out in the pDest-301 Multisite vector (attR4/attR2), which was derived from the pcDNA3.1-zeo vector. The final construct is of the form of CMV3.1p $>A B C B 1>\mathrm{CMV} 13 \mathrm{p}>A B C G 2$, in which the $A B C B 1$ gene is $5^{\prime}$ to the $A B C G 2$ gene, leading to bicistronic production of the two genes from a single expression construct.

After transfection of cells, clones resistant to zeocin were collected, and expression of the various transporters was verified by flow cytometry, as outlined below. Selected clones were maintained in $250 \mu \mathrm{g} / \mathrm{ml}$ zeocin. The positive control HEK293 cell lines transfected with pcDNA vector containing full-length $A B C B 1$ (MDR-19) or $A B C G 2$ (R-5) were previously described (Robey et al., 2003).

Flow Cytometry. Cell surface expression of $\mathrm{ABCB} 1$ and $\mathrm{ABCG} 2$ was verified using phycoerythrin-labeled UIC2 antibody or phycoerythrin-labeled 5D3 antibody (both from Thermo-Fisher), respectively, and corresponding isotype control antibodies. Briefly, cells were trypsinized and incubated with antibodies at saturating concentrations in the absence of substrates or inhibitors
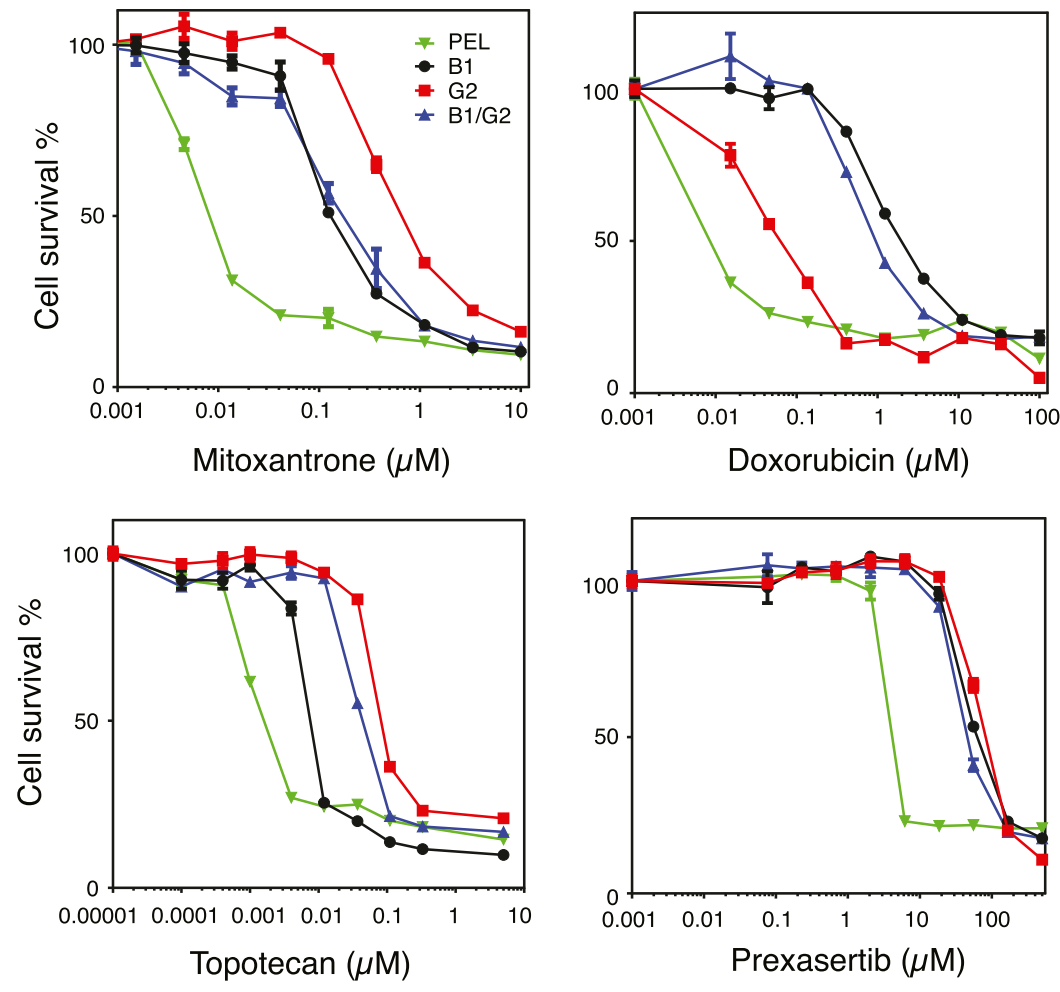

Fig. 3. ABCB1- and ABCG2-expressing cells are resistant to both $\mathrm{ABCB} 1$ and $\mathrm{ABCG} 2$ substrates. Three-day cytotoxicity assays were performed on empty-vector-transfected cells (PEL), $A B C B 1$-transfected cells (B1), ABCG2-transfected cells (G2), or vector containing both genes (B1/G2) with mitoxantrone, doxorubicin, topotecan, and prexasertib. $\mathrm{IC}_{50}$ values were obtained and are summarized in Table 1. 
TABLE 1

Summary of cytotoxicity data with PEL, B1, G2, and B1/G2 cells

Results presented are $I C_{50} \pm S . D$. of at least three independent experiments. Relative resistance $(R R)$ values were determined by dividing the $I C_{50}$ of the transporter-expressing cells by the $I C_{50}$ for the PEL cells.

\begin{tabular}{lccrcrrr}
\hline \multicolumn{1}{c}{ Drug $(\mu \mathrm{M})$} & PEL & B1 & RR & G2 & RR & B1/G2 & RR \\
\hline Mitoxantrone & $0.011 \pm 0.0036$ & $0.17 \pm 0.030$ & 15 & $0.66 \pm 0.35$ & 60 & $0.27 \pm 0.13$ & 24 \\
Doxorubicin & $0.022 \pm 0.011$ & $1.6 \pm 0.50$ & 73 & $0.086 \pm 0.053$ & 4 & $1.2 \pm 0.54$ & 55 \\
Topotecan & $0.0034 \pm 0.0019$ & $0.011 \pm 0.0077$ & 3 & $0.18 \pm 0.078$ & 23 & $0.065 \pm 0.031$ & 19 \\
Prexasertib & $3.7 \pm 0.58$ & $78 \pm 19$ & 21 & $85 \pm 19$ & 23 & $43 \pm 4.6$ & 12
\end{tabular}

in $2 \%$ bovine serum albumin for 20 minutes at room temperature. Cells were washed twice with PBS and then analyzed by flow cytometry.

ABCB1- or ABCG2-mediated transport was examined by a flow cytometry efflux assay (Robey et al., 2004). Trypsinized cells were resuspended in complete medium (phenol red-free Improved Minimum Essential Media with 10\% FBS, $1 \%$ glutamine, and $1 \%$ penicillin) containing $2 \mu \mathrm{M}$ pheophorbide a ( $\mathrm{PhA})$ or $0.5 \mathrm{mg} / \mathrm{ml}$ rhodamine 123 , in the presence or absence of $2.5 \mu \mathrm{M}$ ABCB1 inhibitor VAL (positive control for ABCB1 inhibition), or $10 \mu \mathrm{M}$ ABCG2 inhibitor FTC (positive control for $\mathrm{ABCG} 2$ inhibition), and incubated for 30 minutes at $37^{\circ} \mathrm{C}$ in $5 \% \mathrm{CO}_{2}$. Cells were then washed and incubated for 1 hour at $37^{\circ} \mathrm{C}$ in substratefree complete media continuing with or without VAL or FTC. Subsequently, cells were washed twice with cold PBS and placed on ice until analyzed.

Samples were analyzed by a FACSCanto II flow cytometer (BD Biosciences, San Jose, CA). Phycoerythrin fluorescence was detected by a 488-nm argon laser with a 585-nm bandpass filter. PhA and mitoxantrone fluorescence were detected using a $633-\mathrm{nm}$ HeNe laser with a 660-nm bandpass filter. Rhodamine 123 was
A
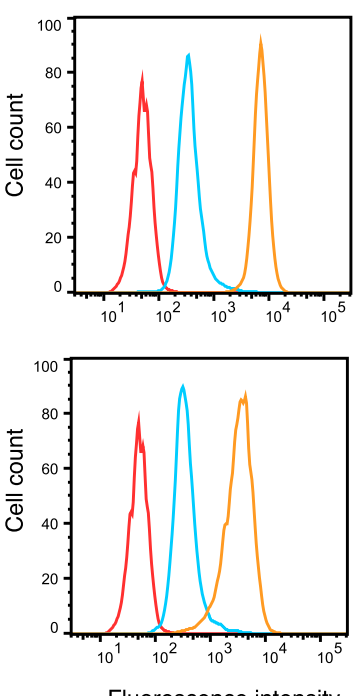

B
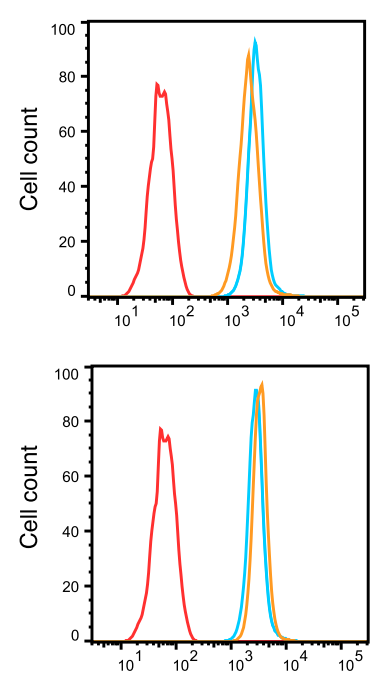

Fluorescence intensity
G2
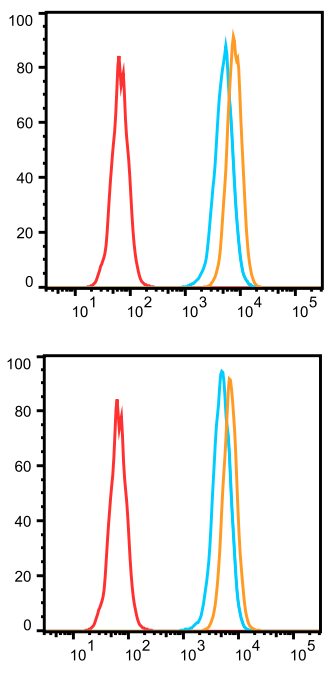

Fluorescence intensity

G2
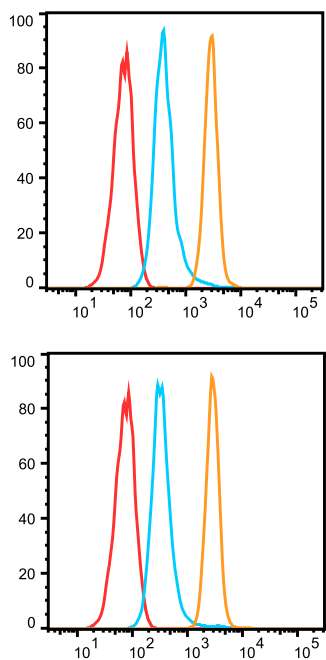

Fluorescence intensity
B1/G2
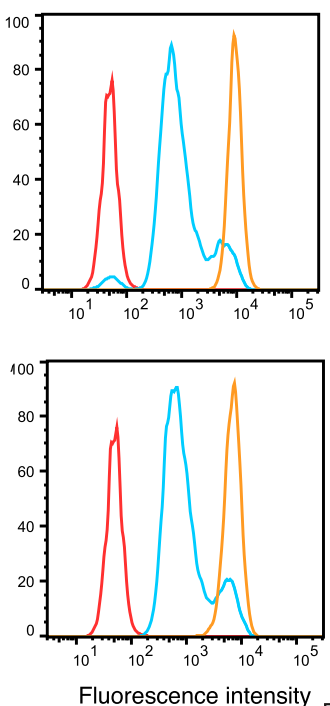

Rho/PhA +

FTC/Nal

$(488 \mathrm{~nm})$

Efflux

- Efflux
- Inhibitor
- Control

$\mathrm{B} 1 / \mathrm{G} 2$

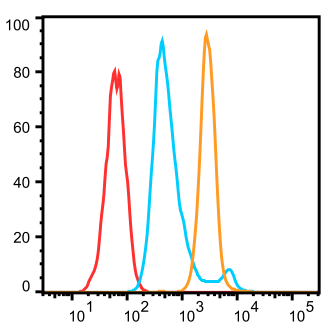

$\mathrm{PhA}+\mathrm{FTC}$

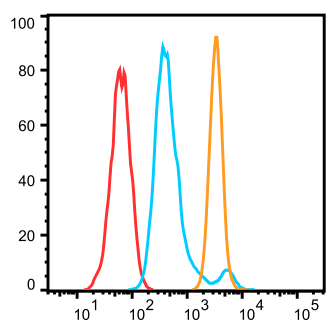

Fluorescence intensity
Rho/Pha +

FTC/Nal

$(650 \mathrm{~nm})$
Fig. 4. $\mathrm{ABCB} 1$ and $\mathrm{ABCG} 2$ can independently transport specific fluorescent substrates. Trypsinized cells were incubated with $0.5 \mu \mathrm{g} / \mathrm{ml}$ rhodamine alone or in combination with $2 \mu \mathrm{m} \mathrm{PhA}$ in the absence or presence of $3 \mu \mathrm{g} / \mathrm{ml} \mathrm{ABCB} 1$ inhibitor VAL, $10 \mu \mathrm{M}$ ABCG2 inhibitor FTC, or both for 30 minutes. Subsequently, cells were incubated with substratefree medium continuing without or with inhibitors for an additional 1 hour. Intracellular fluorescence in the presence of the substrates only is denoted by the orange histogram, whereas fluorescence in the presence of the inhibitors is denoted by the blue histogram. Cell autofluorescence (untreated cells) is denoted by the red histogram. Results from one of three experiments are shown. (A) Top row, cells incubated with rhodamine alone using VAL as the inhibitor; bottom row, cells incubated with rhodamine and PhA simultaneously using both VAL and FTC as the inhibitors. Rhodamine fluorescence was examined using a 488-nm laser. (B) Top row, cells incubated with PhA alone; bottom row, cells incubated with rhodamine and $\mathrm{PhA}$ simultaneously using both VAL and FTC as the inhibitors. PhA fluorescence was examined using a $650-\mathrm{nm}$ laser 
detected with a 488-nm argon laser with a 530-nm bandpass filter. At least 10,000 events were collected for each sample.

Cytotoxicity Assays. Briefly, trypsinized cells were seeded in an opaque white 96-well plate (5000 cells/well) and allowed to attach overnight at $37^{\circ} \mathrm{C}$ in $5 \%$ $\mathrm{CO}_{2}$. Anticancer compounds were added at various concentrations and incubated for 72 hours at $37^{\circ} \mathrm{C}$ in $5 \% \mathrm{CO}_{2}$. Cell viability was determined using CellTiter-Glo (Promega, Madison, WI) reagent, according to the manufacturer's instructions. Luminescence was subsequently read on a Tecan Infinite M200 Pro microplate reader (Tecan Group, Morrisville, NC). Each determination was performed in triplicate.

Immunoblot Analysis. Cell lysates $(30 \mu \mathrm{g})$ were heated to $37^{\circ} \mathrm{C}$ for 20 minutes and then subjected to electrophoresis on a premade $4 \%-12 \%$ bis-tris gel and transferred to a nitrocellulose membrane. Subsequently, the blot was blocked with Odyssey Blocking Buffer (LI-COR, Lincoln, NE) for 1 hour at room temperature and then probed overnight with mouse monoclonal anti-glyceraldehyde-3-phosphate dehydrogenase (1:8000; American Research Products, Waltham, MA), the anti-Pgp antibody C219 (1:250; Signet Laboratories, Dedham, MA), and anti-ABCG2 antibody BXP-21 (1:250; Kamiya Biomedical, Seattle, WA). The blot was then incubated with a goat anti-mouse secondary antibody tagged with a near infra-red fluorochrome, and fluorescence was measured using the LI-COR Odyssey CLx (Li-COR).

\section{Results}

Characterization of a Transfected Cell Line Engineered to Coexpress ABCB1 and ABCG2. Despite the fact that ABCB1 and ABCG2 are coexpressed in many cancers (Robey et al., 2018), few studies have examined the effect that coexpression has on drug resistance and inhibitor efficacy. To that end, we transfected HEK293 cells to express $\mathrm{ABCB} 1$ and $\mathrm{ABCG} 2$ individually as well as together, using a novel bicistronic vector to express both $A B C B 1$ and $A B C G 2$. After transfection, we examined transporter expression in the clones and selected a set in which the cells transfected to express both transporters expressed $\mathrm{ABCB} 1$ and $\mathrm{ABCG} 2$ at levels similar to those of cells that were transfected with the transporter genes individually. Our initial screen of clones examined surface expression by flow cytometry, and we selected a set based on similar staining with the UIC2 and 5D3 antibodies that detected ABCB1 or ABCG2, respectively. Cells expressing both $\mathrm{ABCB} 1$ and ABCG2 (B1/G2) demonstrated similar levels of transporter expression comparable to that of the clones that express ABCB1 (B1) or ABCG2 (G2) individually (Fig. 1A), although levels of both ABCB1 and ABCG2 in $\mathrm{B} 1 / \mathrm{G} 2$ cells were somewhat lower than the individually transfected cell lines. The G2 cell line expresses a low level of ABCB1 that is attributed to endogenous levels in the parental HEK293 cells. Empty vector-transfected cells (PEL) also express low levels of ABCB1 and no ABCG2. Western blot analysis also demonstrated comparable levels of $\mathrm{ABCB} 1$ and $\mathrm{ABCG} 2$ in the double-transfected cell line as compared with cells expressing only one transporter, with empty vector-transfected cells expressing no detectable protein from either transporter (Fig. 1B). MDR-19 and R-5 cells were used as positive controls for $\mathrm{ABCB} 1$ and $\mathrm{ABCG} 2$, respectively, in the immunoblot assay.

To confirm that each of the proteins was functional and capable of transporting substrates, we examined the transport of the fluorescent substrates rhodamine 123 and $\mathrm{PhA}$, specific substrates of $\mathrm{ABCB} 1$ and
B1
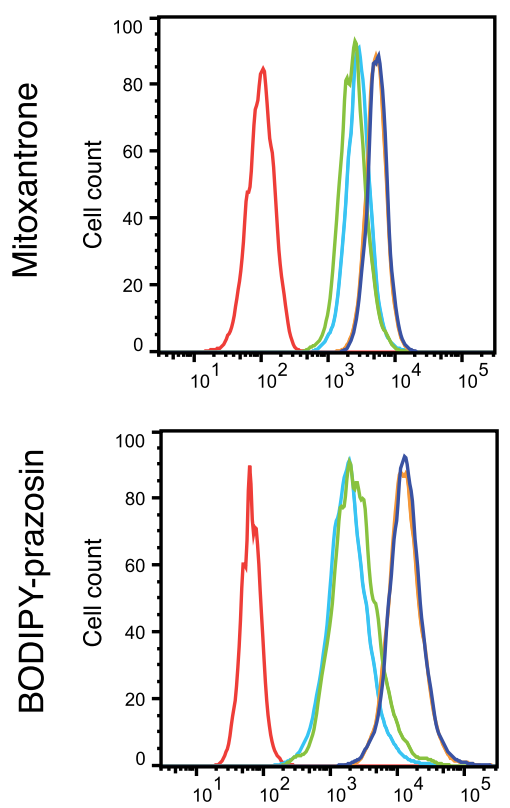

Fluorescence intensity
G2
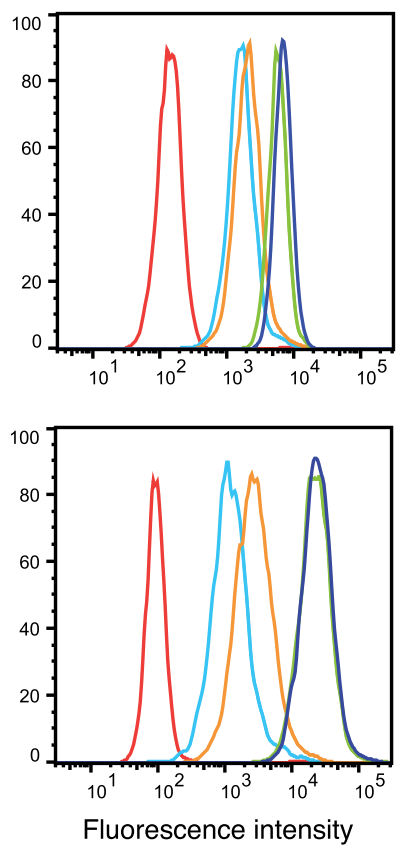

B1/G2
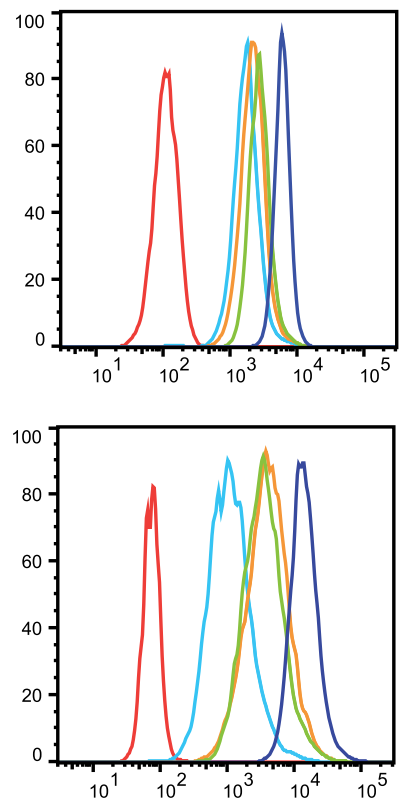

Fluorescence intensity
PEL
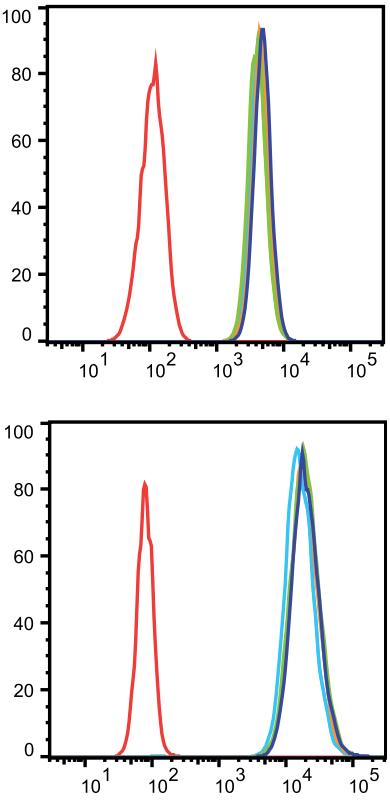

Fluorescence intensity

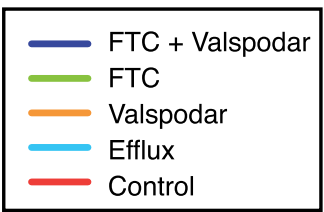

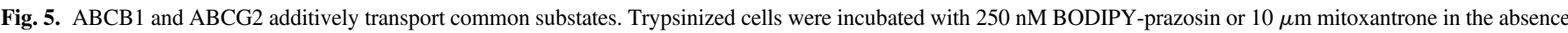

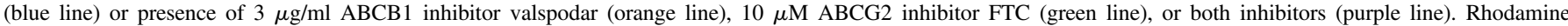

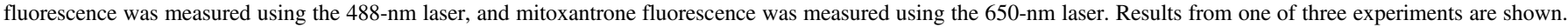


ABCG2, respectively. As can be observed in Fig. 2, B1/G2 cells that coexpress $\mathrm{ABCB} 1$ and $\mathrm{ABCG} 2$ efflux $\mathrm{PhA}$ to a similar degree as the G2 cells that express only ABCG2 (blue histogram). Similarly, B1/G2 cells demonstrate a similar decrease in rhodamine 123 fluorescence as $\mathrm{B} 1$ cells that express only ABCB1. Thus, levels of functional ABCB1 appear to be similar in the B1 and B1/G2 cells, and the levels of functional ABCG2 appear to be similar in the $\mathrm{G} 2$ and $\mathrm{B} 1 / \mathrm{G} 2$ cell lines based on comparable transport of specific substrates.

We also performed 3-day cytotoxicity assays on B1, G2, and B1/G2 cells to verify that $\mathrm{B} 1 / \mathrm{G} 2$ cells were resistant to drugs that are substrates of both ABCB1 and ABCG2. We performed cytotoxicity assays with mitoxantrone, doxorubicin, topotecan, and prexasertib. As shown in Fig. 3, we found that both B1 and B1/G2 cell lines were more resistant to doxorubicin than $\mathrm{G} 2$ cells, that $\mathrm{G} 2$ and $\mathrm{B} 1 / \mathrm{G} 2$ cells were more resistant to topotecan than $\mathrm{B} 1$ cells, and that all transporterexpressing cells displayed high levels of resistance to the common substrate mitoxantrone. We also found that the cell cycle checkpoint kinase 1 inhibitor prexasertib is transported by both ABCB 1 and $\mathrm{ABCG} 2$, as all transporter-expressing cells were resistant to the drug. Relative resistance values are summarized in Table 1. Despite expressing both transporters, B1/G2 cells did not appear to have greater resistance to mitoxantrone or prexasertib than the cells expressing the transporters individually. This is most likely due to the fact that levels of the individual transporters are somewhat lower in B1/G2 cells compared with expression in the cells expressing the transporters individually (see Fig. 1). After verifying that B1/G2 cells express high levels of functional ABCB1 and ABCG2, we performed further experiments on the cells to determine the contribution of each transporter to drug resistance.

ABCB1 and ABCG2 Can Independently Efflux TransporterSpecific Fluorescent Substrates. We next examined whether ABCB1 and ABCG2 can operate independently of each other by examining transport of specific substrates in B1/G2 cells and compared this with transport in B1 and $\mathrm{G} 2$ cells. We performed efflux studies in a manner similar to those done for Fig. 2, except we exposed the B1/G2 cells to both $\mathrm{PhA}$ and rhodamine 123 simultaneously, in the presence or absence of the inhibitors VAL and FTC alone or in combination (FTC/VAL). Substrate efflux was monitored by examining rhodamine fluorescence using the 488-nm laser (488 nm) and $\mathrm{PhA}$ using the 650-nm laser $(650 \mathrm{~nm})$ so as to avoid spectral overlap of the substrates. We also performed the assay with the substrates individually, using VAL with rhodamine 123 (RHO + VAL) and FTC with PhA (PhA + FTC). As can be observed in Fig. 4A, when cells were incubated with rhodamine alone (top row) or $\mathrm{PhA}$ and rhodamine together (second row), comparable rhodamine efflux is observed in the B1 and B1/G2 cells; only a slight amount of efflux is observed in the G2 cells. Furthermore, no transport is observed in B1 cells when cells were incubated with PHA alone (top row) or PhA and rhodamine together (second row), as evidenced by the nearly overlapping blue and orange histograms (Fig. 4B). However, G1 and B1/G2 cells transport PhA similarly, as shown by the comparable distance between the blue and orange histograms (Fig. 4B). These studies demonstrate that B1 and G2 can function independently of one another to transport specific substrates when coexpressed.

For Substrates Common to Both Transporters, Inhibiting Either ABCB1 or ABCG2 When They Are Coexpressed Only Partially Prevents Transport. We next examined the transport of common fluorescent substrates in the B1, G2, and B1/G2 cells. We selected mitoxantrone and BODIPY-prazosin, fluorescent compounds that are transported by both ABCB1 and ABCG2 (Litman et al., 2000; Robey et al., 2008). In this experiment, B1, G2, or B1/G2 cells were incubated with mitoxantrone or BODIPY-prazosin in the presence or
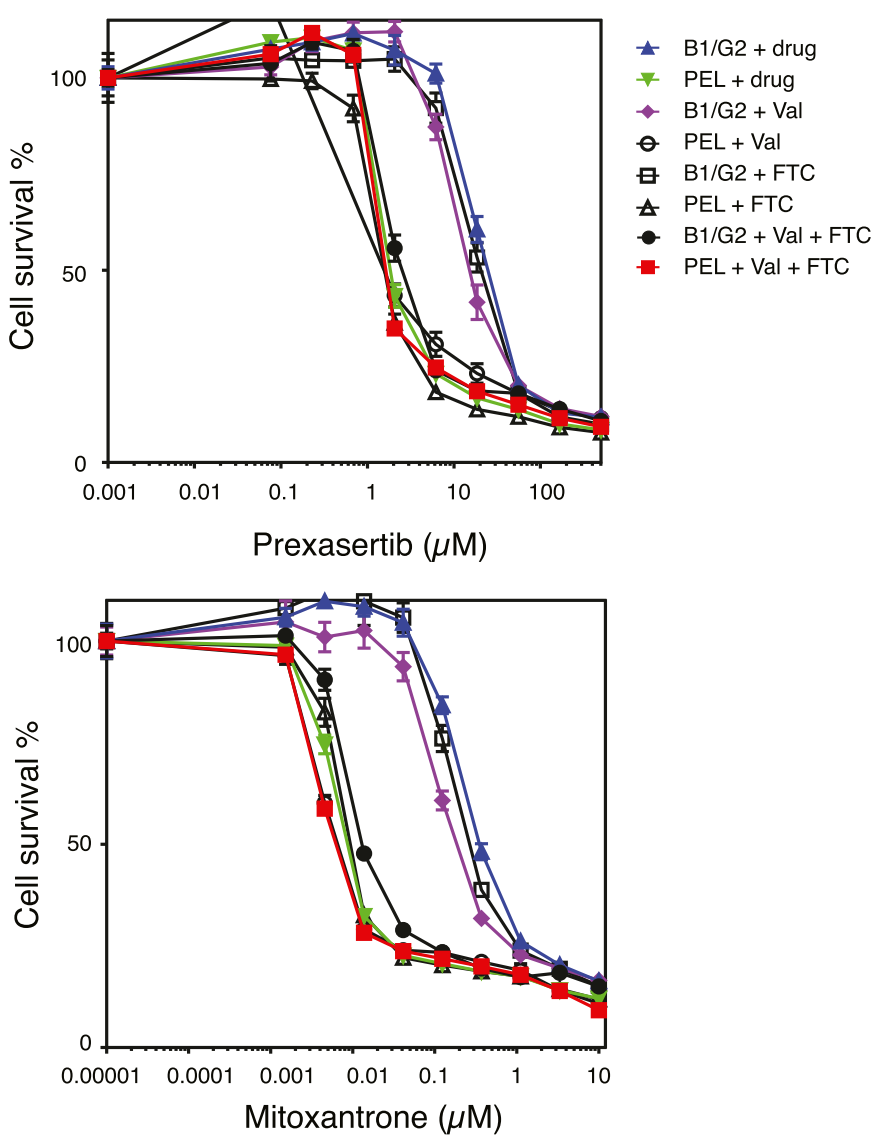

Fig. 6. $\mathrm{ABCB} 1$ and $\mathrm{ABCG} 2$ confer additive resistance to common substrates. Fourday cytotoxicity assays were performed on PEL or B1/G2 cells with mitoxantrone or prexasertib in the absence or presence of $3 \mu \mathrm{g} / \mathrm{ml}$ VAL, $10 \mu \mathrm{M}$ FTC, or both. Results from one of three experiments are shown, and results are summarized in Table 2 .

absence of VAL, FTC, or both inhibitors, as outlined above in Materials and Methods. Results with mitoxantrone or BODIPYprazosin are shown in rows 1 and 2 of Fig. 5, respectively. As observed in the top row of Fig. 5, virtually no efflux is prevented when B1 cells are incubated in mitoxantrone in the presence of FTC (green histogram), and this histogram overlaps with the histogram when cells are incubated in mitoxantrone alone (blue histogram). However, intracellular mitoxantrone fluorescence increases in the presence of VAL (orange histogram), as well as when FTC and VAL are added together with mitoxantrone (purple histogram). In the case of the G2 cells, VAL added to the mitoxantrone (orange histogram) does little to increase fluorescence compared with cells incubated with mitoxantrone alone (blue histogram). Only FTC (green histogram) is able to increase intracellular mitoxantrone levels, and this overlaps with cells incubated with mitoxantrone in the presence of both inhibitors (purple histogram). B1/G2 cells incubated with mitoxantrone in the presence of VAL (orange histogram) or FTC (green histogram) do not appreciably increase fluorescence compared with cells incubated with mitoxantrone alone (blue histogram). Only when B1/G2 cells are incubated with VAL and FTC together (purple histogram) is efflux maximally inhibited.

Similar results were found with BODIPY-prazosin (Fig. 5, bottom row). In B1 cells incubated with BODIPY-prazosin, only VAL (orange histogram) or both VAL and FTC (purple histogram) are able to increase intracellular prazosin levels to levels higher than those of cells incubated with prazosin alone (blue histogram). In the case of G2 
TABLE 2

Summary of cytotoxicity data examining the contribution of ABCB1 and ABCG2 to resistance to mitoxantrone and prexasertib

Results presented are $I C_{50} \pm$ S.D. of at least three independent experiments.

\begin{tabular}{|c|c|c|c|c|c|c|c|c|}
\hline Drug $(\mu \mathrm{M})$ & PEL & PEL + Val & PEL + FTC & $\mathrm{PEL}+\mathrm{Val}+\mathrm{FTC}$ & $\mathrm{B} 1 / \mathrm{G} 2$ & $\mathrm{~B} 1 / \mathrm{G} 2+\mathrm{Val}$ & $\mathrm{B} 1 / \mathrm{G} 2+\mathrm{FTC}$ & $\mathrm{B} 1 / \mathrm{G} 2+\mathrm{Val}+\mathrm{FTC}$ \\
\hline Mitoxantrone & $0.006 \pm 0.002$ & $0.004 \pm 0.0005$ & $0.008 \pm 0.001$ & $0.004 \pm 0.0005$ & $0.3 \pm 0.06$ & $0.1 \pm 0.08$ & $0.2 \pm 0.1$ & $0.01 \pm 0.0005$ \\
\hline Prexasertib & $2.2 \pm 0.043$ & $1.8 \pm 0.16$ & $1.9 \pm 0.094$ & $1.8 \pm 0.12$ & $13 \pm 8.6$ & $12 \pm 2.1$ & $16 \pm 3.5$ & $5.5 \pm 3.9$ \\
\hline
\end{tabular}

cells, VAL (orange histogram) does slightly increase prazosin fluorescence compared with cells incubated with prazosin alone (blue histogram), most likely due to inhibition of the low levels of ABCB 1 expressed by HEK293 cells. However, maximal inhibition is observed when cells are incubated in prazosin with FTC (green histogram) or both VAL and FTC (purple histogram). Finally, in the B1/G2 cells, incubating cells with prazosin in the presence of VAL (orange histogram) or FTC (green histogram) have equal effects on increasing intracellular fluorescence, but maximal inhibition is only observed when both inhibitors are added (purple histogram). These results indicate that both $\mathrm{ABCB} 1$ and $\mathrm{ABCG} 2$ must be inhibited to completely prevent transport of common substrates when both transporters are expressed.

In Cytotoxicity Assays, ABCB1 and ABCG2 Display SubstrateSpecific Additive Activity. To determine the effects of $A B C B 1$ and ABCG 2 coexpression on resistance to long-term exposure to common substrates, 3-day cytotoxicity assays were performed on emptyvector-transfected and B1/G2-transfected clones. We chose mitoxantrone and prexasertib, which are transported by both transporters relatively well, as previously shown. The contribution of each transporter to resistance to the drugs was determined by performing cytotoxicity assays in the absence or presence of VAL and FTC alone or in combination. As shown in Fig. 6, B1/G2 cells are approximately 40-fold more resistant to mitoxantrone compared with B1/G2 cells incubated with both VAL and FTC. When the cells are incubated with mitoxantrone in the presence of the ABCG2 inhibitor FTC, the cells are only about 30-fold more resistant; when the cells are incubated with mitoxantrone in the presence of the ABCB1 inhibitor VAL, the cells are approximately 20-fold more resistant (Table 2). This suggests that the transporters are conferring additive resistance to mitoxantrone. This was confirmed in another B1/G2 clone (F6) (Supplemental Fig. 1A), in which cells were 50 -fold more resistant to mitoxantrone in the absence of inhibitors compared with the presence of both VAL and FTC; cells were 8-fold more resistant to mitoxantrone in the presence of VAL and 33-fold more resistant in the presence of FTC, again suggesting additive resistance (Supplemental Fig. 1B; Supplemental Table 1). The same was true for prexasertib (Fig. 6), as B1/G2 cells were approximately 10-fold more resistant to prexasertib compared with B1/G2 cells incubated with prexasertib in the presence of VAL and FTC. When B1/G2 cells were incubated with prexasertib in the presence of FTC, resistance was 8-fold greater; when the cells were incubated with prexasertib and VAL, resistance was only 6-fold more. Again, we conclude that $\mathrm{ABCB} 1$ and $\mathrm{ABCG} 2$ must both be inhibited to completely overcome resistance to drugs that are substrates of both transporters in cells in which both transporters are expressed.

ABCG2 Expression Decreases Efficacy of the ABCB1 Inhibitor Tariquidar in Cells Expressing Both Transporters. Previous work in our laboratory demonstrated that the $\mathrm{ABCB} 1$ inhibitor tariquidar is a substrate of ABCG2 (Kannan et al., 2011), potentially signifying
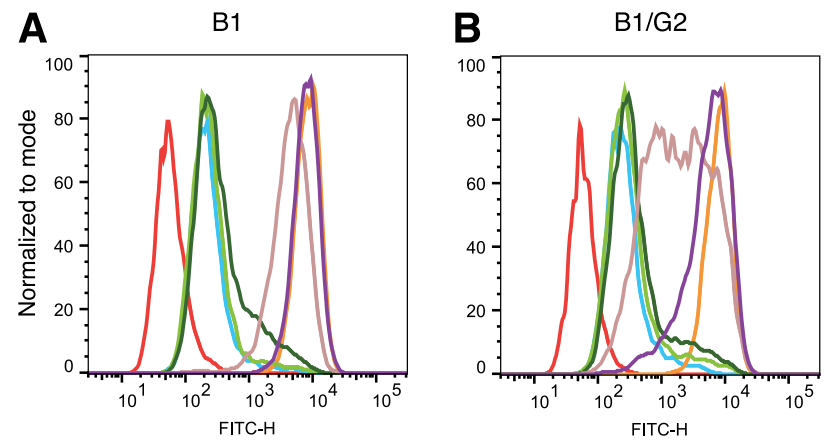

$$
\begin{aligned}
& \text { - Tariquidar, } 250 \mathrm{nM} \\
& \text { - Tariquidar, } 100 \mathrm{nM} \\
& \text { - Tariquidar, } 25 \mathrm{nM} \\
& \text { - Tariquidar, } 10 \mathrm{nM} \\
& \text { - Valspodar } \\
& \text { - Efflux } \\
& \text { - Control }
\end{aligned}
$$

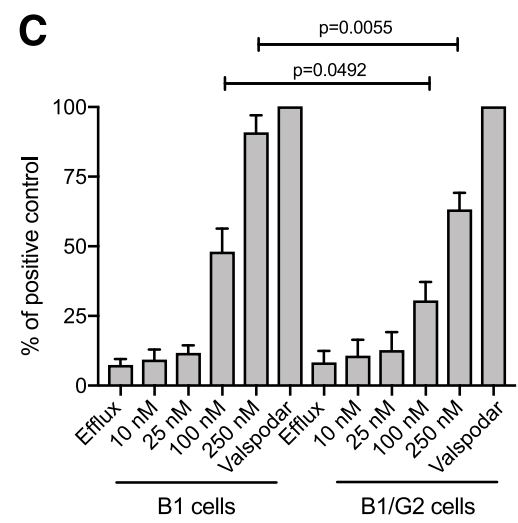

Fig. 7. ABCG2 expression diminishes the ability of tariquidar to inhibit ABCB1. (A) B1 or (B) B1/G2 cells were incubated with $0.5 \mu \mathrm{g} / \mathrm{ml}$ rhodamine 123 in the absence or presence of increasing concentrations of tariquidar $(10,25,100,250 \mathrm{nM})$ for 30 minutes, after which the medium was removed and replaced with substrate-free medium continuing without or with the inhibitor. Results from one of three experiments are shown. (C) Results from the three experiments are summarized and presented as percentage of total inhibition (afforded by $3 \mu \mathrm{M}$ valspodar). 
that coexpression of $\mathrm{ABCG} 2$ could alter the ability of tariquidar to inhibit $\mathrm{ABCB} 1$. We thus examined rhodamine efflux in $\mathrm{B} 1 / \mathrm{G} 2$ cells incubated with increasing amounts of tariquidar and compared this with efflux in $\mathrm{B} 1$ cells that express only ABCB1 to see whether ABCG2 expression had any impact on the ability of tariquidar to inhibit $\mathrm{ABCB} 1$. The ABCG2 inhibitor VAL was used as a positive control. As seen in Fig. 7A, the ability of tariquidar to inhibit $\mathrm{ABCB} 1$ was affected by the presence of $\mathrm{ABCG} 2$, as the ability of tariquidar to inhibit $\mathrm{ABCB} 1$ at the 100 and $250 \mathrm{nM}$ concentrations was different in ABCB1-overexpressing cells (Fig. 7A) and cells expressing both ABCB1 and ABCG2 (Fig. 7B). When the fluorescence of cells incubated with rhodamine in the presence of VAL was assigned a value of $100 \%$, the percent inhibition in cells incubated with rhodamine in the presence of 100 and $250 \mathrm{nM}$ tariquiar was significantly different in B1 and B1/G2 cells (Fig. 7C). Thus, in drug-resistant cancer cells, coexpression of ABCG2 may decrease the ability of tariquidar to inhibit ABCB1.

\section{Discussion}

In this study, we attempted to delineate how ABCB1 and ABCG2 contribute to drug resistance when coexpressed in a cell line model. We used a novel bicistronic vector to develop the B1/G2 cell line that coexpresses both transporters. In the $\mathrm{B} 1 / \mathrm{G} 2$ cell line, we find that $\mathrm{ABCB} 1$ and $\mathrm{ABCG} 2$ are both fully functional and readily transport known substrates. In flow cytometry studies, the transporters are capable of transporting specific substrates such as rhodamine 123 (specific for $\mathrm{ABCB} 1$ ) and $\mathrm{PhA}$ (specific for ABCG2) independently of each other when exposed to the compounds simultaneously, but also act additively to transport common substrates such as BODIPY-prazosin and mitoxantrone. In cytotoxicity assays, the transporters appear to additively confer resistance to common substrates such as mitoxantrone and prexasertib. These results point to the need to inhibit both transporters to overcome resistance to drugs that are substrates of both transporters. Additionally, the transporters more than likely can confer resistance to combination chemotherapy if the components are transporter substrates. We also demonstrate that the presence of ABCG2 can decrease the efficacy of the ABCB1 inhibitor tariquidar. The frequent failure of transporter inhibitors in clinical trials may have been due to their inability to inhibit multiple transporters at clinically achievable concentrations or may be due to another transporter decreasing the efficacy of an inhibitor.

Because we found that expression of multiple transporters led to additive resistance to chemotherapy, it seems reasonable to assume that if multiple transporters were expressed at low levels, they might confer clinically meaningful levels of drug resistance. This was previously demonstrated by Allen et al. (2000), who created a series of mouse embryonic fibroblasts (MEFs) that were proficient or deficient in mouse $A b c b l a / 1 b$ and $A b c c 1$ expression. Compared with wild-type MEFs, MEFs deficient in Abcbla/b expression were 16-fold, 2.4-fold, 4.7-fold, and 2.5-fold more sensitive to paclitaxel, vincristine, doxorubicin, or mitoxantrone, respectively, whereas cells deficient in both $A b c b l a / 1 b$ and $\mathrm{Abccl}$ were 22-fold, 28-fold, 7.1-fold, or 4.3-fold more sensitive to the drugs, respectively (Allen et al., 2000). Thus, if a drug-resistant cell line expressed three different transporters that conferred only 2-fold more resistance to a drug, inhibiting only one might still lead to a cell that was 4-fold more resistant to the compound, which might be clinically meaningful. This is supported by clinical data showing that expression of multiple transporters leads to a worse clinical prognosis (Liu et al., 2018). Inhibition of all transporters expressed by a cancer cell may thus be necessary to overcome transporter-mediated drug resistance.
We also report in this work for the first time that the checkpoint kinase 1 inhibitor prexasertib is transported by both ABCB1 and ABCG2. This is not surprising, as a number of targeted therapies have been shown to interact with one or both of the transporters (Robey et al., 2018). Some of the more recently identified transporter substrates include the fibroblast growth factor receptor AZD4547 (Kas et al., 2018), the DOT1L inhibitor pinometostat (Campbell et al., 2017), the hepatocyte growth factor receptor inhibitor PHA-665752 (Sugano et al., 2015), and the survivin inhibitor YM-155 (Lamers et al., 2012). As most compounds that are substrates of either ABCB1 or ABCG2 generally do not cross the BBB (Durmus et al., 2015a), it does not appear that prexasertib would be able to treat cancer that has metastasized to the brain or primary brain tumors. Clinically achievable concentrations of prexasertib are in the 100-175 nM range (Brill et al., 2017).

Our model does not recapitulate knockout mouse studies in which knockout of both transporters results in greatly increased brain penetration of some chemotherapeutic agents. In the case of axitinib, brain penetration of the drug was not significantly changed in Abcg2-deficient mice compared with wild-type, whereas brain penetration was 4.9-fold higher in mice deficient in Abcb1a/1b and 20.7-fold higher in mice deficient in both transporters compared with wild-type mice at 4 hours after oral administration (Poller et al., 2011a). Similar results were found with the Janus kinase $1 / 2$ inhibitor momelotinib, in which, compared with wild-type mice, mice deficient in Abcg2 had 6.5-fold higher brain levels of momelotinib, mice deficient in $\mathrm{ABcb} 1 \mathrm{a} / 1 \mathrm{~b}$ had 3.1-fold higher levels, and mice deficient in both transporters had 48.3-fold higher levels 8 hours after oral administration of the drug. This suggests a synergistic role for ABCB1 and ABCG2 at the $\mathrm{BBB}$, due to the fact that the effect of the transporters on substrates is greater than passive diffusion across the BBB (Kusuhara and Sugiyama, 2009). That we were not able to mimic the mouse model is most likely due to the fact that HEK293 cells do not form epithelial monolayers with tight junctions that would limit passive diffusion. This is supported by Poller et al. (2011b), who developed a doubly-transduced MDCKII cell line that expresses human $A B C B 1$ and $A B C G 2$. MDCKII cells are known to form monolayers with tight junctions (Brückner and Janshoff, 2018). Poller et al. (2011b) found that the inverse monolayer transport ratios of topotecan, sorafenib, and sunitinib in MDCKII-ABCB1/ABCG2 cells without inhibition, in the presence of Ko143 to inhibit ABCG2, in the presence of zosuquidar to inhibit $\mathrm{ABCB} 1$, or in the presence of both inhibitors, closely modeled in vivo brain penetration of wild-type mice, mice deficient in Abcg2, mice deficient in Abcb1a/1b, or mice deficient in both transporters, respectively (Poller et al., 2011b). Our model would therefore be more applicable to to drug-resistant cancer cells that generally do not form tight junctions and transporter monolayers.

In conclusion, we have generated a cell line model that expresses high levels of human ABCB1 and ABCG2. The transporters appear to function independently as well as additively to confer resistance to substrates. This cell line model may be useful in studying the role of these transporters in cancer drug resistance.

\section{Acknowledgments}

We appreciate the editorial assistance of George Leiman.

\section{Authorship Contributions}

Participated in research design: Robey, Gottesman.

Conducted experiments: Robinson, Tebase, Francone, Huff, Kozlowski, Cossari, Robey.

Contributed new reagents or analytic tools: Lee, Esposito. Performed data analysis: Robinson, Robey, Gottesman.

Wrote or contributed to the writing of the manuscript: Robinson, Robey, Gottesman. 


\section{References}

Allen JD, Brinkhuis RF, van Deemter L, Wijnholds J, and Schinkel AH (2000) Extensive contribution of the multidrug transporters P-glycoprotein and Mrp1 to basal drug resistance. Cancer Res 60:5761-5766.

Brill E, Yokoyama T, Nair J, Yu M, Ahn YR, and Lee JM (2017) Prexasertib, a cell cycle checkpoint kinases 1 and 2 inhibitor, increases in vitro toxicity of PARP inhibition by preventing Rad51 foci formation in BRCA wild type high-grade serous ovarian cancer. Oncotarget 8: 111026-111040.

Brückner BR and Janshoff A (2018) Importance of integrity of cell-cell junctions for the mechanics of confluent MDCK II cells. Sci Rep 8:14117.

Campbell CT, Haladyna JN, Drubin DA, Thomson TM, Maria MJ, Yamauchi T, Waters NJ, Olhava EJ, Pollock RM, Smith JJ, et al. (2017) Mechanisms of pinometostat (EPZ-5676) treatment-emergent resistance in $M L L$-rearranged leukemia. Mol Cancer Ther 16:1669-1679.

Cooray HC, Blackmore CG, Maskell L, and Barrand MA (2002) Localisation of breast cance resistance protein in microvessel endothelium of human brain. Neuroreport 13:2059-2063.

Durmus S, Hendrikx JJ, and Schinkel AH (2015a) Apical ABC transporters and cancer chemotherapeutic drug disposition. Adv Cancer Res 125:1-41.

Durmus S, Sparidans RW, van Esch A, Wagenaar E, Beijnen JH, and Schinkel AH (2015b) Breast cancer resistance protein (BCRP/ABCG2) and P-glycoprotein (P-GP/ABCB1) restrict oral availability and brain accumulation of the PARP inhibitor rucaparib (AG-014699). Pharm Res 32:37-46.

Durmus S, Sparidans RW, Wagenaar E, Beijnen JH, and Schinkel AH (2012) Oral availability and brain penetration of the B-RAFV600E inhibitor vemurafenib can be enhanced by the P-glycoprotein (ABCB1) and breast cancer resistance protein (ABCG2) inhibitor elacridar. Mol Pharm 9:3236-3245.

Durmus S, Xu N, Sparidans RW, Wagenaar E, Beijnen JH, and Schinkel AH (2013) P-glycoprotein (MDR1/ABCB1) and breast cancer resistance protein (BCRP/ABCG2) restrict brain accumulation of the JAK1/2 inhibitor, CYT387. Pharmacol Res 76:9-16.

Fetsch PA, Abati A, Litman T, Morisaki K, Honjo Y, Mittal K, and Bates SE (2006) Localization of the ABCG2 mitoxantrone resistance-associated protein in normal tissues. Cancer Lett 235 : 84-92.

Kannan P, Telu S, Shukla S, Ambudkar SV, Pike VW, Halldin C, Gottesman MM, Innis RB, and Hall MD (2011) The "specific" P-glycoprotein inhibitor tariquidar is also a substrate and an inhibitor for breast cancer resistance protein (BCRP/ABCG2). ACS Chem Neurosci 2:82-89.

Kas SM, de Ruiter JR, Schipper K, Schut E, Bombardelli L, Wientjens E, Drenth AP, de KorteGrimmerink R, Mahakena S, Phillips C, et al. (2018) Transcriptomics and transposon mutagenesis identify multiple mechanisms of resistance to the FGFR inhibitor AZD4547. Cancer Res 78:5668-5679.

Kodaira H, Kusuhara H, Ushiki J, Fuse E, and Sugiyama Y (2010) Kinetic analysis of the cooperation of P-glycoprotein (P-gp/Abcb1) and breast cancer resistance protein (Bcrp/Abcg2) in limiting the brain and testis penetration of erlotinib, flavopiridol, and mitoxantrone. J Pharmacol Exp Ther 333:788-796.

Kusuhara H and Sugiyama Y (2009) In vitro-in vivo extrapolation of transporter-mediated clearance in the liver and kidney. Drug Metab Pharmacokinet 24:37-52.

Lamers F, Schild L, Koster J, Versteeg R, Caron HN, and Molenaar JJ (2012) Targeted BIRC5 silencing using YM155 causes cell death in neuroblastoma cells with low ABCB1 expression. Eur J Cancer 48:763-771.

Litman T, Brangi M, Hudson E, Fetsch P, Abati A, Ross DD, Miyake K, Resau JH, and Bates SE (2000) The multidrug-resistant phenotype associated with overexpression of the new ABC halftransporter, MXR (ABCG2). J Cell Sci 113:2011-2021.

Liu B, Li LJ, Gong X, Zhang W, Zhang H, and Zhao L (2018) Co-expression of ATP binding cassette transporters is associated with poor prognosis in acute myeloid leukemia. Oncol Lett 15: 6671-6677.

Maliepaard M, Scheffer GL, Faneyte IF, van Gastelen MA, Pijnenborg AC, Schinkel AH, van De Vijver MJ, Scheper RJ, and Schellens JH (2001) Subcellular localization and distribution of the breast cancer resistance protein transporter in normal human tissues. Cancer Res 61:3458-3464.

Minocha M, Khurana V, Qin B, Pal D, and Mitra AK (2012) Enhanced brain accumulation of pazopanib by modulating P-gp and Bcrp1 mediated efflux with canertinib or erlotinib. Int $J$ Pharm 436:127-134.
Patel C, Stenke L, Varma S, Lindberg ML, Björkholm M, Sjöberg J, Viktorsson K, Lewensohn R, Landgren O, Gottesman MM, et al. (2013) Multidrug resistance in relapsed acute myeloid leukemia: evidence of biological heterogeneity. Cancer 119:3076-3083.

Poller B, Iusuf D, Sparidans RW, Wagenaar E, Beijnen JH, and Schinkel AH (2011a) Differential impact of P-glycoprotein (ABCB1) and breast cancer resistance protein (ABCG2) on axitinib brain accumulation and oral plasma pharmacokinetics. Drug Metab Dispos 39: 729-735.

Poller B, Wagenaar E, Tang SC, and Schinkel AH (2011b) Double-transduced MDCKII cells to study human P-glycoprotein (ABCB1) and breast cancer resistance protein (ABCG2) interplay in drug transport across the blood-brain barrier. Mol Pharm 8:571-582.

Robey RW, Honjo Y, Morisaki K, Nadjem TA, Runge S, Risbood M, Poruchynsky MS, and Bates SE (2003) Mutations at amino-acid 482 in the ABCG2 gene affect substrate and antagonist specificity. Br J Cancer 89:1971-1978.

Robey RW, Massey PR, Amiri-Kordestani L, and Bates SE (2010) ABC transporters: unvalidated therapeutic targets in cancer and the CNS. Anticancer Agents Med Chem 10:625-633.

Robey RW, Pluchino KM, Hall MD, Fojo AT, Bates SE, and Gottesman MM (2018) Revisiting the role of ABC transporters in multidrug-resistant cancer. Nat Rev Cancer 18:452-464.

Robey RW, Shukla S, Finley EM, Oldham RK, Barnett D, Ambudkar SV, Fojo T, and Bates SE (2008) Inhibition of P-glycoprotein (ABCB1)- and multidrug resistance-associated protein 1 (ABCC1)-mediated transport by the orally administered inhibitor, CBT-1((R)). Biochem Pharmacol 75:1302-1312

Robey RW, Steadman K, Polgar O, Morisaki K, Blayney M, Mistry P, and Bates SE (2004) Pheophorbide a is a specific probe for ABCG2 function and inhibition. Cancer Res 64 1242-1246.

Sparreboom A, van Asperen J, Mayer U, Schinkel AH, Smit JW, Meijer DK, Borst P, Nooijen WJ, Beijnen JH, and van Tellingen O (1997) Limited oral bioavailability and active epithelial excretion of paclitaxel (Taxol) caused by P-glycoprotein in the intestine. Proc Natl Acad Sci USA 94:2031-2035

Sugano T, Seike M, Noro R, Soeno C, Chiba M, Zou F, Nakamichi S, Nishijima N, Matsumoto M, Miyanaga A, et al. (2015) Inhibition of ABCB1 overcomes cancer stem cell-like properties and acquired resistance to MET inhibitors in non-small cell lung cancer. Mol Cancer Ther 14: 2433-2440.

Tang SC, Lagas JS, Lankheet NA, Poller B, Hillebrand MJ, Rosing H, Beijnen JH, and Schinke $\mathrm{AH}$ (2012) Brain accumulation of sunitinib is restricted by P-glycoprotein (ABCB1) and breast cancer resistance protein (ABCG2) and can be enhanced by oral elacridar and sunitinib coadministration. Int J Cancer 130:223-233.

Thiebaut F, Tsuruo T, Hamada H, Gottesman MM, Pastan I, and Willingham MC (1987) Cellular localization of the multidrug-resistance gene product P-glycoprotein in normal human tissues. Proc Natl Acad Sci USA 84:7735-7738.

van Hoppe S, Sparidans RW, Wagenaar E, Beijnen JH, and Schinkel AH (2017) Breast cancer resistance protein (BCRP/ABCG2) and P-glycoprotein (P-gp/ABCB1) transport afatinib and restrict its oral availability and brain accumulation. Pharmacol Res 120:43-50.

Wall VE, Garvey LA, Mehalko JL, Procter LV, and Esposito D (2014) Combinatorial assembly of clone libraries using site-specific recombination. Methods Mol Biol 1116:193-208.

Wilson CS, Davidson GS, Martin SB, Andries E, Potter J, Harvey R, Ar K, Xu Y, Kopecky KJ, Ankerst DP, et al. (2006) Gene expression profiling of adult acute myeloid leukemia identifies novel biologic clusters for risk classification and outcome prediction. Blood 108:685-696.

Yang JJ, Milton MN, Yu S, Liao M, Liu N, Wu JT, Gan L, Balani SK, Lee FW, Prakash S, et al (2010) P-glycoprotein and breast cancer resistance protein affect disposition of tandutinib, a tyrosine kinase inhibitor. Drug Metab Lett 4:201-212.

Zaher H, Khan AA, Palandra J, Brayman TG, Yu L, and Ware JA (2006) Breast cancer resistance protein (Bcrp/abcg2) is a major determinant of sulfasalazine absorption and elimination in the mouse. Mol Pharm 3:55-61.

Address correspondence to: Dr. Michael M. Gottesman, Laboratory of Cell Biology, National Cancer Institute, National Institutes of Health, 37 Convent Drive, Room 2108, Bethesda, MD 20892. E-mail: mgottesman@nih.gov 